\title{
PERFORMANCE EVALUATION OF NUCLEATED RED BLOOD CELL (NRBC) COUNT USING A FULLY AUTOMATED HAEMATOLOGY ANALYZER VERSUS MANUAL COUNTING
}

\author{
Tanweer Ahmed, Asad Mahmood, Nasir Uddin*, Helen Mary Robert, Muhammad Ashraf**, Usman Tahir Swati \\ Armed Forces Institute of Pathology/National University of Medical Sciences (NUMS) Rawalpindi Pakistan, *Combined Military Hospital Lahore/National \\ University of Medical Sciences (NUMS) Pakistan, **Hazrat Bari Imam Sarkar (HBS) Medical and Dental College, Islamabad Pakistan
}

\section{ABSTRACT}

Objective: To evaluate the performance of Nucleated RBC (NRBC) Count using a fully automated haematology analyzer versus manual counting.

Study Design: Cross-Sectional Study.

Place and Duration of Study: Department of Hematology, Armed Forces Institute of Pathology, from Sep 2019-Jun 2020.

Methodology: Routine fresh whole blood samples were run on Sysmex XN-3000 automated haematology analyzer and 384 samples with results of $\geq 0.1 \%$ Nucleated red blood cells were included in this study. Manual NRBC counting was carried out twice on Leishman-stained peripheral blood smears from all 384 samples. Comparison between manual and automated nucleated red blood cell counting methods was statistically analyzed through linear regression analysis \& coefficient correlation. The degree of agreement between two methods was analyzed through Bland-Altman plot. Finally, concordance between the two methods was also analyzed at 5 different ranges of nucleated red blood cells.

Results: Linear regression analysis revealed a $\left(\mathrm{r}^{2}\right)$ value of 0.97 . Regression equation was calculated as $\mathrm{XN}=0.76 \mathrm{MC} \pm 1.28$, with $95 \%$ limits of agreement between $\pm 40.42 \%$ and $-24.47 \%$. A mean bias of $7.97 \%$ was demonstrated through Bland-Altman plot. Concordance analysis revealed a concordance rate of $93.74 \%(360 / 384)$. Nucleated red blood cell counting between two methods were more concordant when nucleated red blood cell counts were $<200 \%$.

Conclusion: Nucleated red blood cells counting by XN-3000 automated hematology analyzer is statistically comparable to manual nucleated red blood cell counting. We suggest that automated counting can be adopted in routine hematology laboratory as a replacement of manual NRBC counting.

Keywords: Automated haematology analyzer, Manual nucleated red blood cell count, Nucleated red blood cell count.

How to Cite This Article: Ahmed A, Mahmood A, Uddin N, Robert HM, Ashraf M, Swati UT. Performance Evaluation of Nucleated Red Blood Cell (NRBC) Count Using A Fully Automated Haematology Analyzer Versus Manual Counting. Pak Armed Forces Med J 2021; 71(5): 1806-1810. Doi: https://doi.org/10.51253/pafmj.v71i5.5706

This is an Open Access article distributed under the terms of the Creative Commons Attribution License (https://creativecommons.org/licenses/by-nc/4.0/), which permits unrestricted use, distribution, and reproduction in any medium, provided the original work is properly cited.

\section{INTRODUCTION}

Nucleated red blood cell (NRBC) or erythroblast is an immature precursor of mature non-NRBC. ${ }^{1}$ In healthy individuals, including neonates beyond first week after birth and non-pregnant women, NRBCs are only found in bone marrow. ${ }^{2}$ NRBCs escaping the marrow into peripheral circulation are rapidly cleared by a functional spleen through its reticuloendothelial and sinusoidal systems. Presence of NRBCs in the peripheral blood is always associated with pathological state and warrant thorough clinicopathological assessment. ${ }^{3}$ Primary pathological mechanisms responsible for appearance of NRBCs in the peripheral blood may include asplenia/hyposplenia, stressed \& ineffective erythropoiesis (thalassemia, megaloblastosis, severe anaemia, myelodysplastic syndromes etc.) and primary hematopoietic abnormalities (e.g., acute leukaemia, chronic leukemias, myelofibrosis etc). ${ }^{4}$ In thalassemia and some

Correspondence: Dr Tanweer Ahmed, Department of Haematology, Armed Forces Institute of Pathology, Rawalpindi Pakistan

Received: 20 Nov 2020; revision received: 24 Feb 2021; accepted: 26 Feb 2021 other hematologic illnesses, burden of NRBCs in peripheral blood carries special significance related to prognosis and therapeutic decisions. ${ }^{5}$ Keeping in view their significance, NRBCs must be accurately detected, enumerated and reported in haematology laboratories. As per recommendations of The International Council for Standardization in Haematology (ICSH), NRBCs are counted and reported as an absolute NRBC count within the differential or the number of NRBCs per 100 White Blood Cells (WBCs) . 6 Presence of significant number of NRBCs in peripheral blood may interfere with WBC count, so a corrected WBC count is calculated and reported. ${ }^{7}$

The most widely used method of NRBC detection and enumeration is manual counting under 400X light microscope after Romanowsky staining of peripheral blood smear. This method is simple but tedious and protracted. It is also inherently associated with imprecision and interobserver variation. ${ }^{8}$ Sysmex XN-3000 and other newer automated haematology analyzers are equipped with NRBC detection and enumeration 
technologies. These analyzers measure NRBCs and other parameters as a routine complete blood count (CBC) without requirement of a separate reagent or software command. These features make automated NRBC counting an affordable and swift method in comparison to manual counting. ${ }^{9}$

Manual counting NRBCs is the most commonly used method in our setups. However, international research has revealed that manual method is imprecise, time-consuming and labor intensive, especially in a hematology laboratory with high volume of samples. Automated method has been researched and validated in many studies and most large laboratories are using this method in routine. This study was deliberated to evaluate the relationship and degree of agreement between manual and automated methods, thus, allowing routine utilization of automated method.

\section{METHODOLOGY}

This cross-sectional study was conducted at Department of Hematology, Armed Forces Institute of Pathology (AFIP), Rawalpindi, from Sep 2019 to Jun 2020. Approval from Institutional Review Board (FCHEM18-1/READ-IRB/20/570) was obtained before starting this study.

Using non-probability consecutive sampling technique, a sample size of 384 was calculated with prevalence of $50 \%$ and confidence interval of $95 \%$.

Inclusion Criteria: Fresh venous whole blood specimens collected from both in-hospital and outpatient departments, of any age group were included.

Exclusion Criteria: Samples from the non-consenting patients were excluded.

Informed written consent was taken from all the participants and parents/guardians of the minors. Fresh venous whole blood was collected from all participants in commercially available EDTA-containing whole blood collection tubes. Each sample was first analyzed by Sysmex XN-3000 automated haematology analyzer within 2 hours of collection and declared fit for inclusion in study if automated NRBC count was $\geq 0.1 \%$. Samples that were analyzed after 2 hours of collection and NRBC count $<0.1 \%$ were excluded from the study.

Sysmex XN-3000 analyzes NRBC in a common WBC \& NRBC (WNR) channel using two reagents: Lysercell WNR haemolysing reagent \& Fluorocell WNR Nucleic acid dye. ${ }^{10}$ Further analysis involves flow cytometric measurements of side fluorescence and forward scatter. Side fluorescence identifies
NRBCs by measuring nucleic acid content, while, forward scatter analyses cell size. Finally, NRBCs are counted and reported as absolute NRBC count as well as percentage NRBC count. ${ }^{11}$

One peripheral whole blood smear was prepared for every sample for manual NRBC count. Romanowsky staining was carried out at room temperature within 2 hours of collection of samples. Staining procedure involved making a smear by spreading a drop of whole blood sample on a clean glass slide by a glassspreader followed by letting it air-dry. The smear was then completely covered with Leishman stain for 2 minutes followed by pouring of $66 \mathrm{mmol} / \mathrm{L}$ Sorensen phosphate buffer at $\mathrm{pH}$ of $6.8 .^{12}$ The slide was then washed in phosphate buffer after 8-10 minutes and placed vertically to drain and dry. Underside of the slide was cleaned and the smear was examined under 400X light microscope by two haematology residents in a doubleblind manner. In case of any significant conflict, an expert haematologist was consulted. Mean NRBC count was calculated from the two counts and reported as number of NRBCs per $100 \mathrm{WBC}$ as per recommendations of ICSH.

Statistical relationship between the manual and automated methods were evaluated by employing linear regression analysis, coefficient of determination $\mathrm{r}^{2}$ ) and regression equation. Bland-Altman analysis was carried out to quantitate the degree of agreement between the two quantitative methods using mean differences, bias between the mean differences and upper \& lower limits of agreement ${ }^{13}$. Regression analysis and Bland-Altman analysis were represented by plots as well. For concordance analysis, six different levels of NRBC ranges were defined: $\leq 1.0 \%, 1.1-10 \%, 10.1-50 \%$, $50.1-100 \%, 100.1-200 \% \& \geq 200.1 \%$. Overall concordance rate and concordance rates at different levels of NRBCs were calculated to view the agreement.

All the data was processed through IBM Statistical Package for Social Sciences (SPSS) version 26 and Microsoft Excel Professional Plus version 2019.

\section{RESULTS}

Age-wise sample distribution revealed that of the total 384 samples, 22 (5.7\%) were from neonates (mean age $5.2 \pm 4.4$ days), $107(27.9 \%)$ were from infants (mean age $6.9 \pm 2.7$ months) and $255(66.4 \%)$ were from individuals from 1-92 years of age (mean age $30.9 \pm$ 21.3 years). Gender-wise sample distribution revealed that $216(56.3 \%)$ samples were from males and 168 $(43.8 \%)$ samples were from females. Majority of individuals with significant NRBC count (i.e. $>5 \%$ ) suffered 
from primary haematologic malignancies followed by thalassemia.

Regression analysis of the data from two methods is shown in Figure-1. A $\left(\mathrm{r}^{2}\right)$ value of 0.97 was highlighted by the analysis. The linear regression equation was calculated as: $\mathrm{XN}=0.76 \mathrm{MC} \pm 1.28$, where $\mathrm{XN}$ is NRBC count (Y-axis) from Sysmex $\mathrm{XN}-3000$, while $\mathrm{MC}$ is manual NRBC count (X-axis) of linear regression plot.

Bland-Altman plot is shown in Figure-II. The mean bias of the data was $7.97 \%$ with $95 \%$ of limits of agreement between upper limit of agreement (ULOA) $40.42 \%$ and lower limit of agreement (LLOA) $-24.47 \%$.

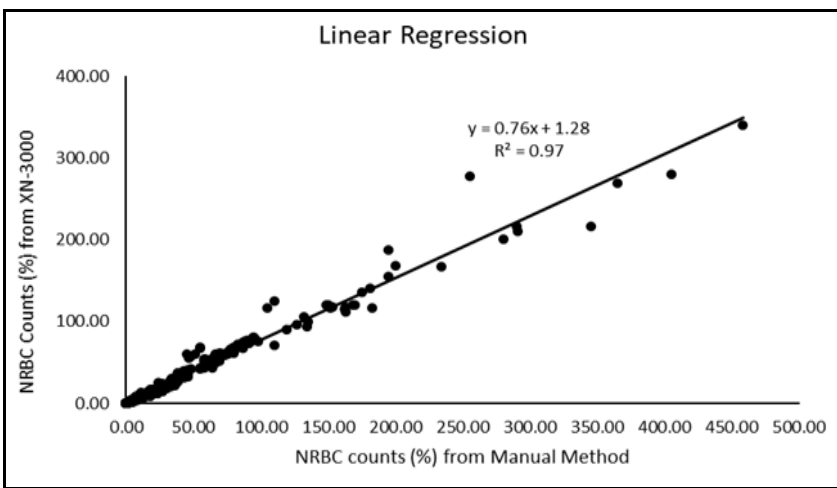

Figure-1: Linear regression analysis of NRBC counts from manual and automated $(\mathrm{XN})$ methods showing $\mathrm{r}^{2}$ \& regression equation.

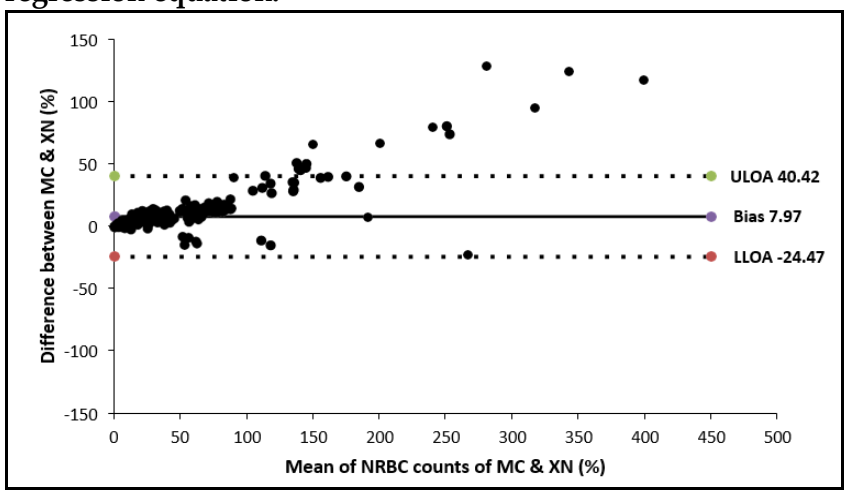

Figure-2: Bland-altman plot showing bias and 95\% limits of agreement.

The agreement of two methods at 06 different levels of NRBCs is shown in the Table. Concordance rate for overall data was $93.74 \%(360 / 384)$. Concordance rates at NRBC count $<200 \%,<100 \%,<50 \%$ and $<10 \%$ were $93.87 \%$ (352/375), 93.28\% (333/352), $96.22 \%$ $(280 / 291)$ and $97.78 \%(176 / 180)$. There was not a single false negative result as per threshold criteria for manual NRBC count of $1 \%$.

\section{DISCUSSION}

The presence of even a single NRBC in the peripheral circulation may be associated with a variety of clinicopathological conditions including severe anaemias, haematologic malignancies, severe hypoxia, sepsis and hyposplenia. ${ }^{14}$ Detection and correct enumeration of NRBCs in peripheral blood may often give clues to these illnesses as well. Therapeutic management and prognosis have also been defined with reference to NRBC count in certain disease like thalassemia. Till today, most of the routine hematologic laboratories detect and report NRBCs using manual method. This method is simple and easy but also depends upon a number of factors for accurate results including staining equipment, technical skill level, expertise of microscopist and may be limited by interobserver variations. At the same time, it adds cost to the diagnostic evaluation. ${ }^{15}$ To cope with these disadvantages, newer automated haematology analyzers have been established that detect and enumerate NRBCs based on side fluorescence and forward scatter principles of flow cytometry. ${ }^{16}$ These analyzers use separate channel for NRBC enumeration. Many studies have demonstrated that these analyzers are fairly precise and accurate. However, these methods carried certain disadvantages like (i) NRBC count are not carried out as part of routine Compete Blood Count (CBC) parameters (ii) NRBC enumeration usually requires separate haemolysing reagents and nucleic acid staining dyes, both adding considerable cost and (iii) Processing time is increased if all samples suspected for NRBCs are run on these analyzers in routine. ${ }^{17}$

Sysmex XN-3000 is a modern state of the art fully automated haematology analyzer that overcomes most of the above-mentioned problems. Based on the same flow cytometric principles, this haematology analyzer

Table: Distribution of nucleated red blood cell count at 6 levels.

\begin{tabular}{|c|c|c|c|c|c|c|c|}
\hline \multicolumn{2}{|c|}{ Manual Nucleated Red Blood Cell Count } & \multirow{2}{*}{\multicolumn{6}{|c|}{$\begin{array}{c}\text { Number of Samples from Sysmex XN-3000 } \\
\text { Range of Nucleated Red Blood Cells (\%) }\end{array}$}} \\
\hline \multirow{2}{*}{$\begin{array}{l}\text { Range of Nucleated } \\
\text { Red Blood Cells (\%) }\end{array}$} & \multirow{2}{*}{$\begin{array}{l}\text { Number of } \\
\text { Samples }\end{array}$} & & & & & & \\
\hline & & $\leq 1.0$ & $1.1-10$ & $10.1-50.0$ & $50.1-100.0$ & $100.1-200.0$ & $\geq 200.1$ \\
\hline$\leq 1.0$ & 56 & 55 & 1 & 0 & 0 & 0 & 0 \\
\hline $1.1-10$ & 124 & 01 & 121 & 02 & 0 & 0 & 0 \\
\hline $10.1-50.0$ & 111 & 0 & 04 & 104 & 03 & 0 & 0 \\
\hline $50.1-100.0$ & 61 & 0 & 0 & 08 & 53 & 0 & 0 \\
\hline $100.1-200.0$ & 23 & 0 & 0 & 0 & 04 & 19 & 0 \\
\hline$\geq 200.1$ & 09 & 0 & 0 & 0 & 0 & 01 & 08 \\
\hline
\end{tabular}


detects and enumerates NRBCs in a common channel that also measures white blood cells. This is especially advantageous as (i) It does not require a separate lysing reagent and staining dye (ii) It carries out NRBC count as part of routine CBC (iii) No re-run of the sample is required for suspicion of presence of NRBCs in the sample. All these advantages result in cost-effectiveness and save turn-around time of the tests. Only a small number of diagnostic centers in Pakistan are equipped with modern fully automated analyzers like Sysmex XN-3000. Literature review revealed that no national study to our knowledge has been published on the evaluation of such analyzers for various parameters of $\mathrm{CBC}$. Our study was aimed at demonstrating relationship and agreeability of the automated method with the traditional manual method of NRBC enumeration.

Our study included samples from individuals who were advised $\mathrm{CBC}$ and peripheral film examination for various clinical and diagnostic purposes. Analysis of our results revealed that NRBC counts from $\mathrm{XN}-3000$ were significantly correlated to those of manual method with $\mathrm{r}^{2}=0.98$. Bland-Altman analysis revealed that overall agreement between automated and manual methods of counting was lacking, however, it yielded a wide range of $95 \%$ upper and lower limits of agreement. The agreement was most deficient when the levels of NRBCs were very high. Concordance analysis between the two methods revealed a very good overall concordance rate of $93.2 \%$ (358/384).

Briggs et al, carried out a very comprehensive study on NRBC count using automated (XN \& XE-2100 modules of Sysmex haematology analyzers) and manual counting methods in 2012. Their study demonstrated an outstanding linear relationship and statistical correlation between automated and manual methods of NRBC counting. ${ }^{18}$ In 2014, Tantanate et al, addressed the performance evaluation of XN-3000 for NRBC counting and compared the results with parallel manual NRBC counting. The study also focused on performance at different levels of NRBCs and concluded that the automated method was agreeable to the manual method when NRBC counts were $<200 \% .{ }^{19}$ Buoro et al, while working on umbilical cord blood, compared two Sysmex modules: XN-modules \& XE-2100 in 2014 and demonstrated a good correlation between Sysmex XNmodule and manual counting. ${ }^{20}$ Da Rin et al, compared performance of five different commercial haematological analyzers including Sysmex XE-2100 and XNmodule. After comparing manual versus automated methods for NRBC count and employing extended statistical methods of evaluation including precision analysis, limit of blank (LoB), limit of detection (LoD) and limit of quantitation (LoQ), the researchers concluded that performance of XN \& XE-2100 modules were excellent. ${ }^{21}$

\section{RECOMMENDATIONS}

Fully automated Sysmex XN-3000 haematology analyzer can be employed for routine NRBC count. More studies on automation and precision analysis of XN-3000 and other automated analyzers are recommended.

\section{CONCLUSION}

Our findings are in accordance with the findings from previous studies with respect to statistical analysis and advantages of employing a fully automated haematology analyzer in routine NRBC count. We suggest that NRBC count by an automated haematology analyzer can replace the manual count in larger haematology laboratories because automation is swift, accurate, and cost-effective. Further research on XN-3000 and other commercially available automated haematology analyzers is strongly recommended.

\section{Conflict of Interest: None.}

\section{Authors' Contribution}

TA: Direct contribution to conception, design, analysis \& interpretation, AM: Intellectual contibution to analysis, literature review \& manuscript preparation, NU: Intellectual contibution to analysis, literature review \& manuscript preparation, HMR: Intellectual contibution to analysis, literature review \& manuscript preparation, MA: Intellectual contribution to analysis, literature review \& manuscript preparation, UTS: Manuscript preparation \& data analysis.

\section{REFERENCES}

1. Purwosunu $Y$, Sekizawa A, Farina A, Okai T, Takabayashi $H$, Wen P, et al. Enrichment of NRBC in maternal blood: a more feasible method for noninvasive prenatal diagnosis. Prenat Diagn 2006; 26(6): 545-547.

2. Esan AJ. Hematological differences in newborn and aging: a review study. Hematol Transfus Int J 2016; 3(3): 178-190.

3. Stachon A, Becker A, Kempf R, Holland-Letz T, Friese J, Krieg M. Re-evaluation of established risk scores by measurement of nucleated red blood cells in blood of surgical intensive care patients. J Trauma 2008; 65(3): 666-673.

4. Danise P, Maconi M, Barrella F, Di Palma A, Avino D, Rovetti A, et al. Evaluation of nucleated red blood cells in the peripheral blood of hematological diseases. Clin Chem Lab Med 2011; 50(2): 357-360.

5. Karakukcu M, Karakukcu C, Unal E, Ozturk A, Ciraci Z, Patiroglu T, et al. The importance of nucleated red blood cells in patients with beta thalassemia major and comparison of two automated systems with manual microscopy and flow cytometry. Clin Lab 2015; 61(9): 1289-1295.

6. Palmer L, Briggs C, McFadden S, Zini G, Burthem J, Rozenberg $\mathrm{G}$, et al. ICSH recommendations for the standardization of nomenclature and grading of peripheral blood cell morphological features. Int J Lab Hematol 2015; 37(3): 287-303. 


\section{Nucleated Red Blood Cell Count}

7. Constantino BT, Rivera GKQ. Cutoff value for correcting white blood cell count for nucleated red blood cells: what is it? Why is it Important. Lab Med 2019; 50(4): e82-e90.

8. Hwang DH, Dorfman DM, Hwang DG, Senna P, Pozdnyakova O. Automated nucleated RBC measurement using the sysmex XE-5000 hematology analyzer: frequency and clinical significance of the nucleated RBCs. Am J Clin Pathol 2016; 145(3): 379-384.

9. Genc S, Dervisoglu E, Erdem S, Arslan O, Aktan M, Omer B. Comparison of performance and abnormal cell flagging of two automated hematology analyzers: Sysmex XN 3000 and Beckman Coulter DxH 800. Int J Lab Hematol 2017; 39(6): 633-640.

10. Kaido M, Takagi Y, Kono M, Nakazawa F, Yamamoto S, Wada A, et al. Investigation of morphological changes for the discrimination of nucleated red blood cells and other leukocytes in Sysmex XN hematology analyzer scattergrams using transmission electron microscopy. Pract Lab Med 2017; 8(5): 70-76.

11. Seo JY. Performance evaluation of the new hematology analyzer Sysmex XN-series. Int J Lab Hematol 2015; 37(2): 155-164.

12. Bain BJ. Preparation and staining methods for blood and bone marrow films. In: Bain BJ, Laffan MA, Bates I. Practical Haematology, $12^{\text {th }}$ edition. Elsevier Limited 2017; 1(1): 50-60.

13. Giavarina D. Understanding Bland Altman analysis. Biochem Med (Zagreb) 2015; 25(2): 141-151.

14. Lehnhardt M, Katzy Y, Langer S, Druecke D, Homann $\mathrm{HH}_{\text {, }}$ Steinstraesser L, et al. Prognostic significance of erythroblasts in burns. Plast Reconstr Surg 2005; 115(1): 120-127.
15. Gulati G, Behling E, Kocher W. An evaluation of the performance of Sysmex XE-2100 in enumerating nucleated red cells in peripheral blood. Arch Pathol Lab Med 2007; 131(7): 10771083.

16. Kwon MJ, Nam MH, Kim SH, Lim CS, Lee CK, Cho Y, et al. Evaluation of the nucleated red blood cell count in neonates using the Beckman Coulter UniCel DxH 800 analyzer. Int J Lab Hematol 2011; 33(6): 620-628.

17. de Keijzer $\mathrm{MH}$, van der Meer W. Automated counting of nucleated red blood cells in blood samples of newborns. Clin Lab Haematol 2002; 24(6): 343-345.

18. Briggs C, Longair I, Kumar P, Singh D, Machin SJ. Performance evaluation of the Sysmex haematology XN modular system. J Clin Pathol 2012; 65(11): 1024-1030.

19. Tantanate C, Klinbua C. Performance evaluation of the automated nucleated red blood cell enumeration on Sysmex XN analyser. Int J Lab Hematol 2015; 37(3): 341-345.

20. Buoro S, Vavassori M, Pipitone S, Benegiamo A, Lochis E, Fumagalli $S$, et al. Evaluation of nucleated red blood cell count by Sysmex XE-2100 in patients with thalassaemia or sickle cell anaemia and in neonates. Blood Transfus 2015; 13(4): 588594.

21. Da Rin G, Vidali M, Balboni F, Benegiamo A, Borin M, Ciardelli $\mathrm{ML}$, et al. Performance evaluation of the automated nucleated red blood cell count of five commercial hematological analyzers. Int J Lab Hematol 2017; 39(6): 663-670. 